\title{
Function and Regulation of Yeast Ribonucleotide Reductase: Cell Cycle, Genotoxic Stress, and Iron Bioavailability
}

\author{
Nerea Sanvisens, Rosa de Llanos, Sergi Puig
}

\begin{abstract}
Ribonucleotide reductases (RNRs) are essential enzymes that catalyze the reduction of ribonucleotides to desoxyribonucleotides, thereby providing the building blocks required for de novo DNA biosynthesis. The RNR function is tightly regulated because an unbalanced or excessive supply of deoxyribonucleoside triphosphates (dNTPs) dramatically increases the mutation rates during DNA replication and repair that can lead to cell death or genetic anomalies. In this review, we focus on Saccharomyces cerevisiae class Ia RNR as a model to understand the different mechanisms controlling RNR function and regulation in eukaryotes. Many studies have contributed to our current understanding of RNR allosteric regulation and, more recently, to its link to RNR oligomerization. Cells have developed additional mechanisms that restrict RNR activity to particular periods when dNTPs are necessary, such as the $\mathrm{S}$ phase or upon genotoxic stress.
\end{abstract} These regulatory strategies include the transcriptional control of the RNR gene

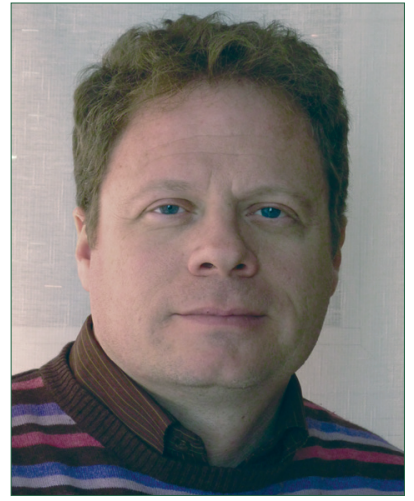

Prof. Sergi Puig expression, inhibition of RNR catalytic activity, and the subcellular redistribution of RNR subunits. Despite class Ia RNRs requiring iron as an essential cofactor for catalysis, little is known about RNR function regulation depending on iron bioavailability. Recent studies into yeast have deciphered novel strategies for the delivery of iron to RNR and for its regulation in response to iron deficiency. Taken together, these studies open up new possibilities to explore in order to limit uncontrolled tumor cell proliferation via RNR.

(Biomed J 2013;36:51-58)

\section{Key words: cell cycle, genotoxic stress, iron deficiency, ribonucleotide reductase, Saccharomyces cerevisiae, yeast}

A 11 eukaryotic organisms require an adequate, balanced concentration of deoxyribonucleoside triphosphates (dNTPs) in order to assure accurate DNA replication and repair, and to maintain genomic integrity. The rate-limiting step in dNTP synthesis is catalyzed by ribonucleotide reductase (RNR), an essential enzyme mediating the reduction of ribonucleotides to desoxyribonucleotides, thereby providing the building blocks required for DNA synthesis. Consistent with its important role in cell proliferation, a significant increase in RNR activity has been associated with tumor cells and resistance to chemotherapy. Indeed, since the utilization of hydroxyurea in the 70 s to the current development of sophisticated RNR inhibitors, RNR as been used as an important target for the chemotherapeutic treatment of numerous cancer types. ${ }^{[1]}$ Therefore, understanding the molecular mechanisms that cells utilize to regulate RNR function in response to different stresses is critical for the development of new and efficient anticancer therapies. In this review, we focus on the yeast S. cerevisiae as a eukaryotic model to advance in our understanding of mechanisms regulating the function of eukaryotic RNRs during cell cycle progress and in response to environmental cues, including genotoxic stress and low iron bioavailability.

\section{RNR structure, assembly, and allosteric regulation}

In eukaryotes, class Ia RNRs are oxygen-dependent enzymes composed of a large R1 $\left(\alpha_{2}\right)$ and a small R2 $\left(\beta_{2}\right.$ or $\left.\beta \beta^{\prime}\right)$ subunit. The $\mathrm{R} 1$ subunit contains the catalytic site and two allosteric effector binding sites that

From the Department of Biotechnology, Institute of Agrochemistry and Food Technology, Spanish Research Council, Valencia, Spain Received: Jul. 25, 2012; Accepted: Oct. 5, 2012

Correspondence to: Prof. Sergi Puig, Department of Biotechnology, Institute of Agrochemistry and Food Technology, Spanish Research Council, Valencia, Spain. Ave. Agustín Escardino 7, Paterna, Valencia, E-46980, Spain. Tel: 34-963-900022 ext. 2308 ;

Fax: 34-963-636301; E-mail: spuig@iata.csic.es

DOI: $10.4103 / 2319-4170.110398$ 
determine substrate preference and overall activity. The R2 subunit harbors a stable diferric tyrosyl radical cofactor $\left(\mathrm{Fe}^{3+}{ }_{2} \mathrm{Y} \cdot\right)$ required for catalysis. Briefly, substrate binding to $\mathrm{R} 1$ initiates a $\sim 35 \AA$ proton-coupled electron transfer pathway from $\mathrm{R} 1$ to the $\mathrm{Fe}^{3+}{ }_{2}-\mathrm{Y}$ in $\mathrm{R} 2$ that leads to ribonucleotide reduction. At each catalytic site, the regeneration of an active RNR requires the reduction of a conserved pair of oxidized cysteines in $\mathrm{R} 1$ by thioredoxin or glutaredoxin. ${ }^{[2-4]}$

One unique feature of $S$. cerevisiae RNR relies on the heterodimeric $\beta \beta^{\prime}$ nature of its active small R2 subunit, composed of two different proteins denoted Rnr2 and Rnr4. Whereas Rnr2 contains the indispensable $\mathrm{Fe}^{3+}{ }_{2}-\mathrm{Y}$ · cofactor, the structurally homologous Rnr4 lacks essential ligands for iron binding and cannot, therefore, form the canonical tyrosyl radical. ${ }^{[5-8]}$ Despite this situation, deletion of $R N R 4$ leads to lethality or severe growth impairment, which highlights its important role in the RNR function. ${ }^{[5,6]}$ Interestingly, in vitro and in vivo studies suggest that Rnr4 contributes to the correct folding and assembly of the $\mathrm{Fe}^{3+}{ }_{2}-\mathrm{Y} \cdot$ cofactor in Rnr2, although the underlying mechanism remains unknown $^{[9-13]}$ (for a recent review on cofactor assembly into class I RNRs, see Ref. 4). Recent evidence demonstrates that conserved cytosolic monothiol glutaredoxins Grx3 and Grx4 function in delivering iron to multiple iron-containing proteins, including Rnr $2 .{ }^{[14]}$ Consistently with this, the yeast cells depleted of Grx 4 exhibit reduced RNR-specific activity due to the inefficient incorporation of iron into Rnr2. ${ }^{[14]}$ Furthermore, depletion of $\mathrm{Fe}-\mathrm{S}$ cluster protein Dre2 also diminishes both Y levels and RNR activity, suggesting that Dre 2 functions in providing reducing equivalents in order to deliver iron in its reduced state. ${ }^{[13]}$ These results, and the genetic interactions among mutants $R N R 4$, grx3 grx4 and Dre2, suggest a model in which Grx3-Grx4 and Dre 2 collaborate in delivering iron ion in its reduced state for $\mathrm{Fe}^{3+}{ }_{2} \mathrm{Y} \cdot$ cluster formation in the Rnr2 partner of the Rnr2-Rnr4 heterodimer. ${ }^{[13]}$

The RNR large subunit is composed of an Rnr1 homodimer, which is essential for mitotic viability ${ }^{[15]} \mathrm{In}$ addition to RNR1, yeast cells express a second RNR large subunit, called $R N R 3$, at very low levels, whose deletion does not cause any phenotype, but whose overexpression rescues the lethality of $R N R 1$ null mutants. ${ }^{[15,16]}$ Despite low Rnr3 specific activity, experiments with a catalytically inactive $R N R 1$ mutant have indicated that Rnr3 increases its endogenous activity by associating with Rnr1. ${ }^{[16]}$ However, further studies are required to elucidate the physiological function of yeast Rnr3.

Sophisticated allosteric regulations in the R1 large subunit contribute to maintain a balanced pool of dNTPs, which is essential to guarantee DNA synthesis fidelity. ${ }^{[17]}$ Each R1 monomer contains two distinct regulatory sites:
The specificity site (or S site) and the activity site (or A site). The $\mathrm{S}$ site, which is located at the dimer interface, acts as a sensor of each dNTP's individual concentration and determines which substrate is reduced at the catalytic site. When the allosteric effector binds to the $\mathrm{S}$ site, it alters the conformation of a flexible loop, which transmits the specific signal to the catalytic site to make it more amenable to discriminate between substrates, thus maintaining a balance among the four dNTP pools. Therefore, adenosine triphosphate (ATP) and deoxyadenosine triphosphate (dATP) promote the reduction of cytidine diphosphate (CDP) and uridine diphosphate (UDP), deoxythymidine triphosphate (dTTP) increases the guanosine diphosphate (GDP) reduction rate, and deoxyguanosine triphosphate (dGTP) up-regulates adenosine diphosphate (ADP) reduction. The A site, which is located in a small ATP amino-terminal cone domain, acts as a master switch, which controls overall enzyme activity by monitoring the dATP (inhibitor)/ATP (activator) ratio. Despite ATP being more abundant in the cell, dATP has a higher affinity for the A site. dATP displays 10-20 times lower affinity for the A site than for the S site. Thus, at concentrations below micromolar, dATP only functions as an S site regulator. When the dATP pool reaches higher concentrations, RNR activity is turned off by dATP feedback inhibition. However, an unusual relaxed dATP feedback inhibition of yeast Rnr1 leads to an expansion of yeast dNTP pools in response to DNA damage, which does not occur in mammalian cells. ${ }^{[18]}$ Lack of Rnr3 inhibition by dATP suggests that an Rnr1-Rnr3 heterodimer may be advantageous in DNA-damaged cells, as it allows larger dNTP pools to accumulate; however, no decrease in dNTPs is observed in $r n r 3 \Delta$ mutants upon DNA damage. ${ }^{[16,18]}$

Studies on mammalian and yeast cells suggest that without nucleotide effectors, R1 exists as an inactive $\alpha$ monomer. Upon the binding of ATP, dATP, thymidine triphosphate (TTP), or dGTP to the S site, R1 forms $\alpha_{2}$ dimers, which can assemble as active $\alpha_{2} \beta_{2}\left(\alpha_{2} \beta \beta^{\prime}\right.$ in yeast) heterotetramers. ${ }^{[19]}$ Structural and functional data indicate that the binding of dATP to the A site promotes the association of three $\alpha_{2}$ dimers to form an $\alpha_{6}$ hexamer, which can interact only with a single $\mathrm{R} 2$ subunit to form an inactive $\alpha_{6} \beta_{2}$ complex ( $\alpha_{6} \beta \beta^{\prime}$ in yeast). ${ }^{[20]}$ According to this model, the conformational changes accompanying dATP-induced hexamerization probably disrupt the proper interaction between the R1 and R2 subunits, leading to the disruption of the protein-coupled electron transport chain. On the other hand, when dATP levels decrease, ATP binding to the A site may promote structurally different conformational changes in the $\alpha_{6} \beta_{2}$ complex, which increase RNR catalytic activity. Additional RNR oligomeric structures, including active $\alpha_{6} \beta_{6}$ complexes, have also been proposed. ${ }^{[17]}$ 


\section{Regulation of RNR during the cell cycle and in response to genotoxic stress}

In all eukaryotic cells, including yeast, RNR activity is tightly controlled in order to avoid undesirable increases in dNTP pools, which raise mutation rates. Thus, the up-regulation of RNR activity is highly restricted to particular periods when dNTPs are required. The Mec1-Rad53-Dun1 DNA damage and replication checkpoint kinase cascade is responsible for promoting yeast RNR activity in response to DNA damage, DNA replication stress, or when cells enter the $\mathrm{S}$ phase of the cell cycle. The Mec1 kinase sensor activates the Rad53 central transducer. Both $M E C 1$ and RAD53 are essential genes that can be rescued only by increasing dNTP pools. Hyperphosphorylated Rad53 activates the Dun1 effector kinase by phosphorylation. Then, the Dun1 checkpoint kinase enhances the RNR function by multiple mechanisms [Figure 1a].

One mechanism involves Crt1, also known as Rfx1, a DNA-binding protein that recognizes 13-nucleotide long cis-regulatory elements known as X-box motives, present in the promoter of damage-inducible genes, including $R N R 2, R N R 3$, and $R N R 4$, and represses their transcription by recruiting the general repressor complex Tup1-Ssn6. ${ }^{[21]}$ The robust repression of $R N R$ genes by the Crt1 protein is achieved by a synergic corepression with Rox 1 and Mot3, two Ssn6-Tup1-recruiting proteins that repress hypoxic genes, including $R N R 2-4 .^{[22]}$ Detailed studies on the nucleosomal structure of the RNR3 promoter have shown that the Ssn6-Tup1 corepressor requires the collaboration of the ISW2 complex to establish a regular array of positioned nucleosomes that represses the $R N R 3$ expression. ${ }^{[23,24]}$ In response to DNA damage or replication blocking, Crt1 becomes hyperphosphorylated via the Mec1-Rad53-Dun1 signaling pathway and moves away from DNA, thus preventing the formation of the repressor complex and leading to the activation of damage-inducible genes [Figure 1a]. ${ }^{[21]}$ Genetic data indicate that $C R T 10$ functions as a positive regulator in the $C R T 1$ pathway in response to DNA damage. ${ }^{[25]}$ Despite the Rox1-Mot3-mediated repression still being functional during genotoxic stress, a substantial part of its repression is lost from lack of a synergy with Crt1.$^{[22]}$ Before dissociating from the RNR3 promoter, Crt1 recruits the Transcription Factor IID (TFIID), the SWItch/ Sucrose NonFermentable SWI/SNF chromatin remodeling complex, and histone deacetylases to promote transcription activation. ${ }^{[26,27]} \mathrm{An}$ additional level of regulation is possible because of the $\mathrm{X}$-box motifs present in the $C R T 1$ promoter permitting an autoregulatory mechanism on the $C R T 1$ gene expression, which mediates its induction upon genotoxic stress and rapidly restores its repressed state after eliminating damage. ${ }^{[21]}$

Regulation of $R N R$ genes upon genotoxic stress is not completely dependent on Dun1 kinase. For instance in dun $1 \Delta$ mutants, $R N R$ genes continue to be significantly induced in response to DNA damage. ${ }^{[21]}$ Furthermore, recent results have demonstrated that the RNRI expression is induced upon DNA damage in a Dun1- and Crt1-independent manner. Instead, in response to genotoxic stress, Mec1 and Rad53 activate Ixr1, a DNA-binding protein that interacts with the RNRI promoter and which activates its transcription $^{[28]}$ [Figure 1a]. Consistently with a reduced $R N R 1$ expression, IXR1 deletion results in lower dNTP levels, sensitivity to DNA damage agents, a Mec1-Rad53Dun1-Crt1-dependent elevation of Rnr3 and Rnr4, and the down-regulation of Sml1 levels, which explain why DUN1 is indispensible in $i r x 1 \Delta$ mutants. ${ }^{[28]}$

The transcriptional regulation of $R N R$ genes also contributes to fluctuating RNR activity during the cell cycle. Whereas RNRI mRNA levels increase more than tenfold in the G1/S phase, $R N R 2$ transcripts display only a slight twofold up-regulation. ${ }^{[15]}$ No cell cycle regulation has been observed for $R N R 3$ and $R N R 4$ transcripts. ${ }^{[5,15]} R N R 1$ transcriptional activation during the transition from G1 to $\mathrm{S}$ phase is regulated by the MBF transcription factor, which coordinately controls the expression of many genes required for early cell cycle functions, including DNA replication and repair. MBF is an heterodimeric complex composed of regulatory transactivating protein Swi6, which is tethered to $\mathrm{MCB}$ elements at the promoter of $R N R 1$ and to other $\mathrm{G} 1 / \mathrm{S}$ genes via its DNA-binding partner, Mbp1 [Figure 1b]. ${ }^{[29,30]}$ The MBF complex restricts the expression of its target genes to the G1/S transition. A Swi6-interacting protein, denoted Stb1, mediates MBF-dependent transcriptional repression, probably via Sin3, prior to its $\mathrm{Cln} / \mathrm{Cyclin}$-Dependent Kinase (Cln/CDK)-dependent phosphorylation, which occurs when cells enter G1. ${ }^{[31-33]}$ In a late G1 phase, Stb1 is released from MBF promoters by an unknown mechanism, while a protein called Nrm1 accumulates, interacts with MBF, and promotes MBF-dependent transcriptional repression in the $\mathrm{S}$ phase. ${ }^{[34]}$ Interestingly, recent results have demonstrated that in the $\mathrm{S}$ phase, genotoxic stress induces the activation of the Rad53 checkpoint kinase, which directly phosphorylates the Nrm1 corepressor in a Dun1-independent manner and prevents it binding to MBF, thereby permitting the specific transcriptional activation of G1/S cell cycle targets, including RNR1..$^{[35,36]}$ Moreover, the cell cycle regulatory SBF complex, composed of Swi6, and DNA-binding protein Swi4 have also been implicated in the transcriptional activation of $R N R 2$ and $R N R 3$ genes in response to DNA damage. ${ }^{[37]}$

As a result of the relaxed dATP feedback inhibition of RNR, yeast cells have developed an additional RNR repression mechanism. The Sml1 protein binds through its carboxy-terminal tail to the yeast R1 large subunit by inhib- 


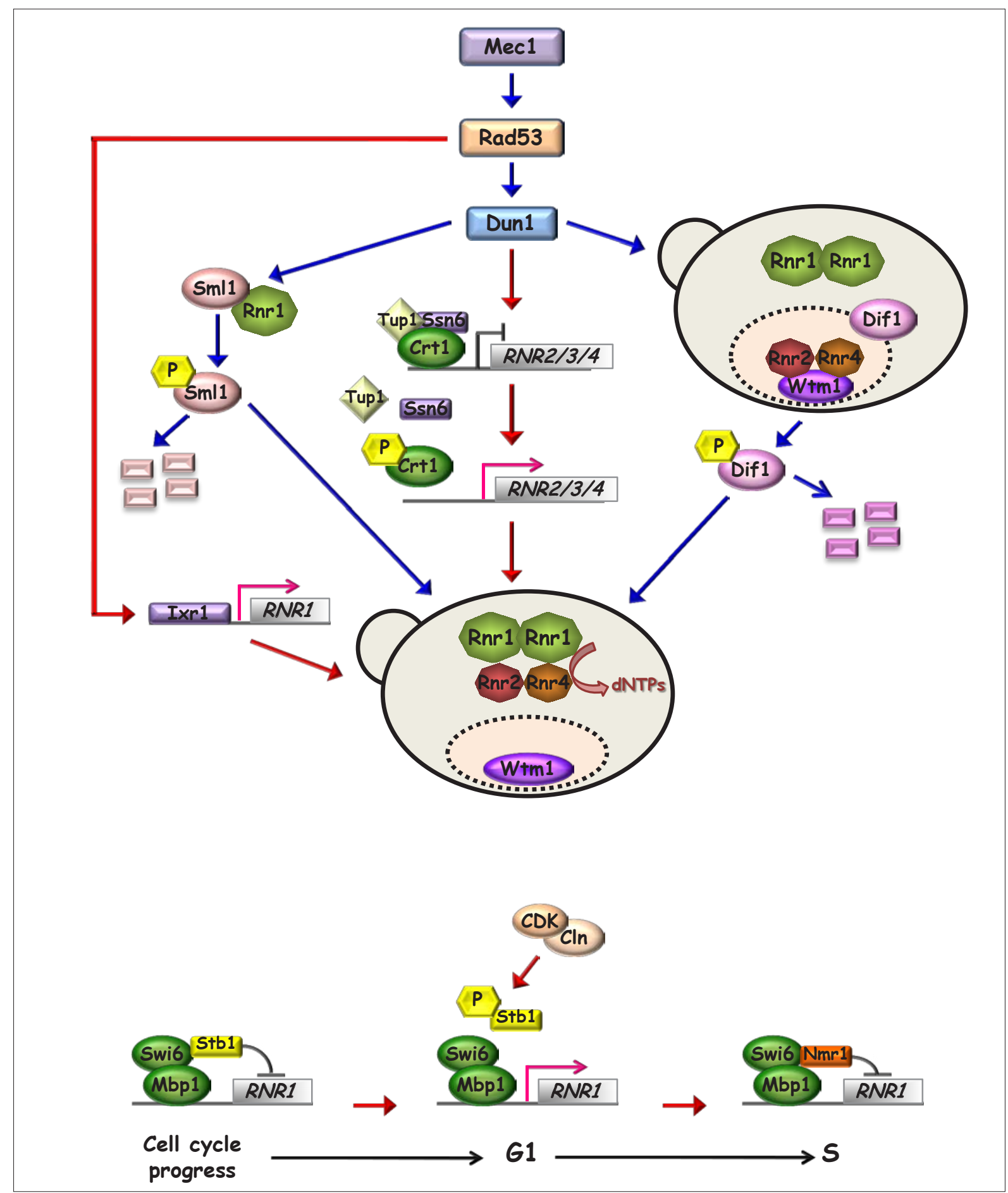

Figure 1: Model for regulating RNR activity in the $\mathrm{S}$ phase and in response to genotoxic stress. In addition to allosteric regulation mechanisms, RNR is tightly regulated in the S phase and after DNA damage or DNA replication blocks. (a) In response to genotoxic stress and in the S phase, the Mec1-Rad53-Dun1 kinase pathway activates RNR activity by (1) promoting the phosphorylation and degradation of the Sml1 R1-inhibitor and (2) enhancing R2 relocalization to the cytoplasm via Wtm1 and Dif1 modifications. Upon genotoxic stress, the Mec1Rad53-Dun1 checkpoint pathway phosphorylates Ctr1, leading to the derepression of $R N R 2 / 3 / 4$ transcription. Recent results show that $R N R 1$ transcription is activated via Ixr1 in a Mec1-Rad53-dependent, but Dun1-independent manner, in response to genotoxic stress. Blue arrows refer to the pathways activated in genotoxic stress and in the $\mathrm{S}$ phase, whereas red arrows indicate the pathways only activated in response to genotoxic stress. (b) The transcription of RNR1 in the G1/S cell cycle transition phase is regulated by the MBF heterodimeric complex, which is composed of the regulatory transactivating protein Swi6 and its DNA-binding partner Mbp1. Several proteins participate in the repression of RNR1 outside the G1/S phase. Stb1 mediates MBF-dependent transcriptional repression prior to its Cln/CDK-dependent phosphorylation occurring at G1, whereas Nrm1 promotes the MBF-dependent transcriptional repression of RNR1 as cells exit the G1 phase 
iting its activity when DNA synthesis is not required. ${ }^{[38-40]}$ Elegant in vivo experiments have shown that when each catalytic cycle is completed, reduction of cysteine residues at the R1 active site in the amino-terminal part of an $\alpha$ protomer is performed by a couple of cysteines located in the carboxy-terminal domain of the other $\alpha$ protomer. ${ }^{[41]}$ Since Sml1 also binds to the amino-terminal region of $\mathrm{R} 1$, it competes with the carboxy-terminal cysteines and inhibits cysteine reduction at the active site and, hence, the enzyme turnover ${ }^{[41]}$ In the $\mathrm{S}$ phase, in response to DNA lesions, Sml1 protein levels dramatically lower, leading to an increase in dNTP levels. The underlying mechanism involves the activation of the checkpoint signaling pathway, which results in the direct phosphorylation of Sml1 by the Dun 1 kinase. ${ }^{[42-45]} \mathrm{Sml1}$ phosphorylation triggers its release from the R1 subunit and its degradation by the $26 \mathrm{~S}$ proteasome to allow RNR activation [Figure 1a]. Recent data have demonstrated that the targeted destruction of phosphorylated Sml1 depends on a multimeric complex which includes E2 ubiquitin-conjugating enzyme Rad6, E3 ubiquitin ligase Ubr2, and E2-E3-interacting protein Mub1. ${ }^{[44]}$

Another mode of RNR regulation is the differential localization of its subunits. Under normal conditions, the yeast R1 large subunit localizes to the cytoplasm, whereas the R2 small subunit is predominantly localized to the nucleus. Upon DNA damage, replication blockage, or in the $\mathrm{S}$ phase, the majority of the $\mathrm{R} 2$ subunit is redistributed from the nucleus to the cytoplasm, resulting in an active RNR complex [Figure 1a]. ${ }^{[46]}$ Intriguingly, R2 redistribution to the cytoplasm is fully dependent on the Mec1-Rad53Dun1 kinase pathway upon treatment with methyl methanesulfonate (MMS), but is only partially dependent when hydroxyurea (HU) is added. ${ }^{[46]}$ In any case, both proteins Rnr2 and Rnr4 in R2 are cotransported between the nucleus and the cytoplasm as one heteromeric complex. ${ }^{[47]}$ The subcellular localization of the R2 small subunit is simultaneously controlled by two different proteins: Wtm1 and Dif1. Under normal growth conditions, the WD40-containing protein, Wtm1, physically interacts with the $\mathrm{R} 2$ subunit by anchoring it to the nucleus. When cells undergo genotoxic stress, this interaction diminishes and the Rnr2-Rnr4 heterodimer relocalizes from the nucleus to the cytoplasm [Figure 1a]. ${ }^{[48,49]}$ The nature of this interaction and the underlying release mechanism upon DNA damage have not been characterized to date. The subcellular localization of $\mathrm{R} 2$ is also regulated by Dif1, a primary cytoplasmic protein containing a Hug domain conserved in Spd1, a Schizosaccharomyces pombe protein that interacts and sequesters the fission yeast R2 subunit into the nucleus away from its catalytic subunit. ${ }^{[50]}$ Under normal conditions, Dif1 binds directly to R2 via the Hug domain and drives its import to the nucleus. ${ }^{[51,52]}$ Dif1 protein levels are regulated during the cell cycle with a maximum increase at the end of the $S$ phase when the R2 small subunit returns to the nucleus. ${ }^{[51]}$ In response to DNA damage or replicative stress, the Dun1 checkpoint kinase directly phosphorylates Dif1 at specific residues within its Sml domain and promotes its degradation relieving RNR inhibition. ${ }^{[31,52]}$ Thus, compartmentalization of the R2 small subunit contributes to modulate RNR activity and dNTP pools to ensure accurate, efficient DNA synthesis.

\section{Regulation of RNR in response to low iron bioavailability}

Iron is an indispensable micronutrient for all eukaryotic organisms because it participates as an essential cofactor in class Ia RNRs, among other reasons. The oxo-diiron center in RNR is responsible for generating and maintaining the stable tyrosyl radical that initiates the electron transfer leading to ribonucleoside diphosphate reduction. The low solubility of ferric iron at physiological $\mathrm{pH}$ frequently leads to iron deficiency, which is the most common and widespread nutritional disorder worldwide. ${ }^{[53]}$ Despite this, very little is known about the mechanisms regulating the RNR function in response to iron limitation. Previous studies in mammals have shown that severe iron deficiency conditions can result in decreased RNR activity and dNTP pools. ${ }^{[54,55]}$ A careful analysis of those studies shows that dNTP synthesis slightly increases during early iron deficiency stages, ${ }^{[55]}$ suggesting that cells may possess mechanisms to maintain the RNR function when iron becomes scarce. By using budding yeast, we recently uncovered a novel mechanism to promote the RNR function when iron availability decreases. ${ }^{\left[{ }^{[6]}\right.} \mathrm{We}$ observed that in response to low iron, yeast $\mathrm{R} 2$ subunits redistribute from the nucleus to the cytoplasm, where $\mathrm{R} 1$ subunits permanently reside, in a manner that is independent of the well-established regulatory activation mechanism controlled by checkpoint kinases Mec1 and Rad53. Instead, a novel strategy promotes the Rnr2-Rnr4 subcellular relocalization, and optimizes both the RNR function and dNTP synthesis when iron bioavailability becomes limited. In response to low iron, yeast cells activate the expression of two RNA-binding proteins, denoted as Cth1 and Cth2, which specifically interact with the AU-rich elements within the $3^{\prime}$ untranslated region of many of the mRNAs encoding proteins which either contain iron or participate in metabolic pathways that utilize iron as a cofactor to promote their degradation. ${ }^{[57,58]}$ Specifically, Cth1 and Cth2 mediate the degradation of transcripts that function in the tricarboxylic acid cycle, mitochondrial electron transport chain, lipid and amino acid metabolisms, and heme synthesis, among other 
iron-dependent pathways. ${ }^{[57,58]}$ Multiple approaches demonstrate that upon iron deficiency, Cth 1 and $\mathrm{Cth} 2$ promote the down-regulation of WTM1 mRNA. ${ }^{[56]}$ The consequent decrease in Wtm1 protein levels facilitates the translocation of Rnr2-Rnr4 to the cytoplasm, leading to dNTP synthesis [Figure 2]. Mutagenesis of either Cth1-Cth2 or WTM1-specific binding motifs reduces $\mathrm{R} 2$ redistribution and diminishes dNTP pools during iron deficiency. ${ }^{[56]}$ These results suggest that the Sml1-mediated repression of the R1 subunit should be relieved under low iron. However, the mechanisms regulating Sml1 under these conditions have not yet been described. Moreover, Cth1 and Cth 2 also interact with $R N R 2$ and $R N R 4$ transcripts in response to iron deficiency by promoting their degradation and limiting new R2 subunit synthesis ${ }^{[56]}$ probably due to the scarce availability of the iron cofactor to incorporate into Rnr2. Interestingly, recent studies in yeast indicate that eukaryotic DNA polymerases rely on iron-sulfur clusters as essential cofactors for the formation of active complexes, thus highlighting the central role of iron in DNA synthesis. ${ }^{[59]}$ Furthermore, a recent study in fission yeast has shown a direct link between DNA synthesis and RNR regulation. Briefly, the interaction of Spd1 with the polymerase processivity factor proliferating cell nuclear antigen (PCNA), complexed onto DNA, is essential for the Spd1 ubiquitylation and degradation that leads to RNR activation. ${ }^{[60]}$ Further studies are required to elucidate how mammalian RNR activity and DNA synthesis are regulated in accordance with iron availability, and how these regulatory mechanisms influence the utilization of RNR as a target for anticancer treatments.

\section{Conclusion}

The budding yeast $S$. cerevisiae has outstandingly contributed to our current understanding of how eukaryotic cells function. Yeast cells possess multiple mechanisms to tightly control RNR activity in response to changes during

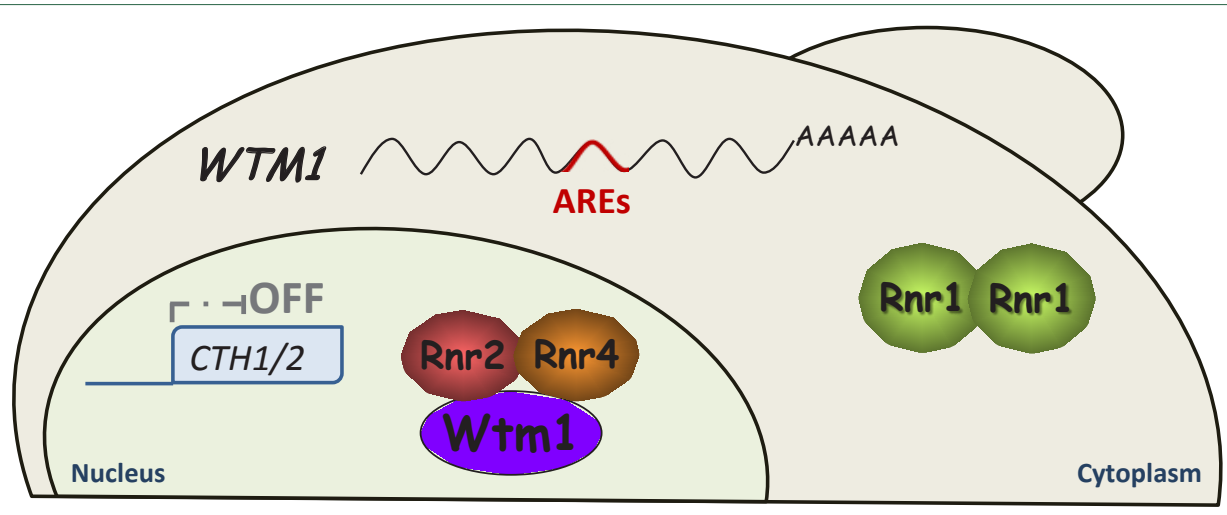

\section{Iron sufficiency}

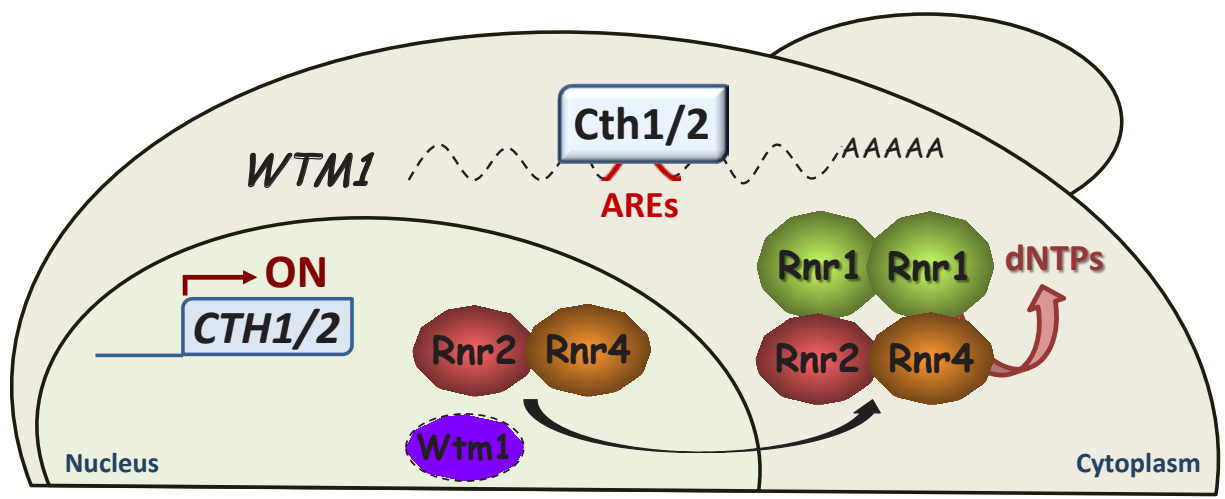

Iron deficiency

Figure 2: Regulation of RNR activity in response to iron deficiency. RNR activity is partially regulated by controlling the subcellular localization of the Rnr2-Rnr4 heterodimer. Under sufficient iron conditions, the catalytic Rnr1 homodimer resides in the cytoplasm, while iron-containing heterodimer Rnr2-Rnr4 is predominantly localized in the nucleus. Wtm1, a WD40 protein, anchors Rnr2-Rnr4 to the nucleus and limits RNR activity. Iron deficiency activates the expression of Cth1 and Cth2 proteins, which bind to WTM1 mRNA and promote its degradation. The drop in the Wtm1 protein levels leads to the Rnr2-Rnr4 subunit translocation to the cytoplasm, where it binds to Rnr1 to form an active RNR enzyme 
the cell cycle and environmental cues such as genotoxic stress and iron deficiency. The time has now come to decipher how these multilayered strategies are interconnected in order to regulate the yeast enzyme and to what extent they occur in mammalian cells.

\section{Acknowledgments}

We apologize to those whose work has not been cited due to space limitations. Nerea Sanvisens is recipient of a predoctoral fellowship from the "Generalitat Valenciana" and Rosa de Llanos is supported by a postdoctoral JAE-Doc contract from the Spanish Research Council (CSIC) and the European Social Fund. Work in our group is supported by the AGL2011-29099 grant from the Spanish Ministry of Economy and Competitiveness to Sergi Puig.

\section{REFERENCES}

1. Richardson DR, Kalinowski DS, Lau S, Jansson PJ, Lovejoy DB. Cancer cell iron metabolism and the development of potent iron chelators as anti-tumour agents. Biochim Biophys Acta 2009;1790:702-17.

2. Kolberg M, Strand KR, Graff P, Andersson KK. Structure, function, and mechanism of ribonucleotide reductases. Biochim Biophys Acta 2004;1699:1-34.

3. Nordlund P, Reichard P. Ribonucleotide reductases. Annu Rev Biochem 2006;75:681-706.

4. Cotruvo JA, Stubbe J. Class I ribonucleotide reductases: Metallocofactor assembly and repair in vitro and in vivo. Annu Rev Biochem 2011;80:733-67.

5. Huang M, Elledge SJ. Identification of RNR4, encoding a second essential small subunit of ribonucleotide reductase in Saccharomyces cerevisiae. Mol Cell Biol 1997;17:6105-13.

6. Wang PJ, Chabes A, Casagrande R, Tian XC, Thelander L, Huffaker TC. Rnr4p, a novel ribonucleotide reductase small-subunit protein. Mol Cell Biol 1997;17:6114-21.

7. Elledge SJ, Davis RW. Identification and isolation of the gene encoding the small subunit of ribonucleotide reductase from Saccharomyces cerevisiae: DNA damage-inducible gene required for mitotic viability. Mol Cell Biol 1987;7:2783-93.

8. Hurd HK, Roberts CW, Roberts JW. Identification of the gene for the yeast ribonucleotide reductase small subunit and its inducibility by methyl methanesulfonate. Mol Cell Biol 1987;7:3673-7.

9. Chabes A, Domkin V, Larsson G, Liu A, Graslund A, Wijmenga S, et al. Yeast ribonucleotide reductase has a heterodimeric iron-radical-containing subunit. Proc Natl Acad Sci USA 2000;97:2474-9.

10. Nguyen HH, Ge J, Perlstein DL, Stubbe J. Purification of ribonucleotide reductase subunits $\mathrm{Y} 1, \mathrm{Y} 2, \mathrm{Y} 3$, and $\mathrm{Y} 4$ from yeast: Y4 plays a key role in diiron cluster assembly. Proc Natl Acad Sci USA 1999;96:12339-44.

11. Voegtli WC, Ge J, Perlstein DL, Stubbe J, Rosenzweig AC. Structure of the yeast ribonucleotide reductase Y2Y4 heterodimer. Proc Natl Acad Sci USA 2001;98:10073-8.
12. Perlstein DL, Ge J, Ortigosa AD, Robblee JH, Zhang Z, Huang M, et al. The active form of the Saccharomyces cerevisiae ribonucleotide reductase small subunit is a heterodimer in vitro and in vivo. Biochemistry 2005;44:15366-77.

13. Zhang Y, Liu L, Wu X, An X, Stubbe J, Huang M. Investigation of in vivo diferric tyrosyl radical formation in Saccharomyces cerevisiae Rnr2 protein: Requirement of Rnr4 and contribution of Grx3/4 and Dre2 proteins. J Biol Chem 2011;286:41499-509.

14. Muhlenhoff U, Molik S, Godoy JR, Uzarska MA, Richter N, Seubert A, et al. Cytosolic monothiol glutaredoxins function in intracellular iron sensing and trafficking via their bound iron-sulfur cluster. Cell Metab 2010;12:373-85.

15. Elledge SJ, Davis RW. Two genes differentially regulated in the cell cycle and by DNA-damaging agents encode alternative regulatory subunits of ribonucleotide reductase. Genes Dev 1990;4:740-51.

16. Domkin V, Thelander L, Chabes A. Yeast DNA damage-inducible Rnr3 has a very low catalytic activity strongly stimulated after the formation of a cross-talking Rnr1/Rnr3 complex. J Biol Chem 2002;277:18574-8.

17. Hofer A, Crona M, Logan DT, Sjoberg BM. DNA building blocks: Keeping control of manufacture. Crit Rev Biochem Mol Biol 2012;47:50-63.

18. Chabes A, Georgieva B, Domkin V, Zhao X, Rothstein R, Thelander L. Survival of DNA damage in yeast directly depends on increased dNTP levels allowed by relaxed feedback inhibition of ribonucleotide reductase. Cell 2003;112:391-401.

19. Rofougaran R, Vodnala M, Hofer A. Enzymatically active mammalian ribonucleotide reductase exists primarily as an $\alpha_{6} \beta_{2}$ octamer. J Biol Chem 2006;281:27705-11.

20. Fairman JW, Wijerathna SR, Ahmad MF, Xu H, Nakano R, Jha $\mathrm{S}$, et al. Structural basis for allosteric regulation of human ribonucleotide reductase by nucleotide-induced oligomerization. Nat Struct Mol Biol 2011;18:316-22.

21. Huang M, Zhou Z, Elledge SJ. The DNA replication and damage checkpoint pathways induce transcription by inhibition of the Crt1 repressor. Cell 1998;94:595-605.

22. Zaim J, Speina E, Kierzek AM. Identification of new genes regulated by the Crt1 transcription factor, an effector of the DNA damage checkpoint pathway in Saccharomyces cerevisiae. J Biol Chem 2005;280:28-37.

23. Zhang Z, Reese JC. Ssn6-Tup1 requires the ISW2 complex to position nucleosomes in Saccharomyces cerevisiae. EMBO J 2004;23:2246-57.

24. Li B, Reese JC. Ssn6-Tup1 regulates $R N R 3$ by positioning nucleosomes and affecting the chromatin structure at the upstream repression sequence. J Biol Chem 2001;276:33788-97.

25. Fu Y, Xiao W. Identification and characterization of CRT10 as a novel regulator of Saccharomyces cerevisiae ribonucleotide reducatese genes. Nucleic Acids Res 2006;34:1876-83.

26. Zhang Z, Reese JC. Molecular genetic analysis of the yeast repressor Rfx 1/Crt1 reveals a novel two-step regulatory mechanism. Mol Cell Biol 2005;25:7399-411.

27. Sharma VM, Tomar RS, Dempsey AE, Reese JC. Histone deacetylases RPD3 and HOS2 regulate the transcriptional activation of DNA damage-inducible genes. Mol Cell Biol 2007;27:3199-210.

28. Tsaponina $\mathrm{O}$, Barsoum E, Astrom SU, Chabes A. Ixr1 is required for the expression of the ribonucleotide reductase Rnr1 and maintenance of dNTP pools. PLoS Genet 2011;7:e1002061. 
29. Koch C, Moll T, Neuberg M, Ahorn H, Nasmyth K. A role for the transcription factors Mbp1 and Swi4 in progression from G1 to $\mathrm{S}$ phase. Science 1993;261:1551-7.

30. Iyer VR, Horak CE, Scafe CS, Botstein D, Snyder M, Brown PO. Genomic binding sites of the yeast cell-cycle transcription factors SBF and MBF. Nature 2001;409:533-8.

31. de Bruin RA, Kalashnikova TI, Wittenberg C. Stb1 collaborates with other regulators to modulate the G1-specific transcriptional circuit. Mol Cell Biol 2008;28:6919-28.

32. Ho Y, Costanzo M, Moore L, Kobayashi R, Andrews BJ. Regulation of transcription at the Saccharomyces cerevisiae start transition by Stb1, a Swi6-binding protein. Mol Cell Biol 1999;19:5267-78.

33. Costanzo M, Schub O, Andrews B. G1 transcription factors are differentially regulated in Saccharomyces cerevisiae by the Swi6-binding protein Stb1. Mol Cell Biol 2003;23:5064-77.

34. de Bruin RA, Kalashnikova TI, Chahwan C, McDonald WH, Wohlschlegel J, Yates $\mathrm{J}, 3^{\text {rd }}$, et al. Constraining G1-specific transcription to late $\mathrm{G} 1$ phase: The MBF-associated corepressor Nrm1 acts via negative feedback. Mol Cell 2006;23:483-96.

35. Travesa A, Kuo D, de Bruin RA, Kalashnikova TI, Guaderrama M, Thai $\mathrm{K}$, et al. DNA replication stress differentially regulates G1/S genes via Rad53-dependent inactivation of Nrm1. EMBO J 2012;31:1811-22

36. Bastos de Oliveira FM, Harris MR, Brazauskas P, de Bruin RA, Smolka MB. Linking DNA replication checkpoint to MBF cell-cycle transcription reveals a distinct class of $\mathrm{G} 1 / \mathrm{S}$ genes. EMBO J 2012;31:1798-810.

37. Ho Y, Mason S, Kobayashi R, Hoekstra M, Andrews B. Role of the casein kinase I isoform, Hrr25, and the cell cycle-regulatory transcription factor, $\mathrm{SBF}$, in the transcriptional response to DNA damage in Saccharomyces cerevisiae. Proc Natl Acad Sci USA 1997;94:581-6.

38. Zhao X, Muller EG, Rothstein R. A suppressor of two essential checkpoint genes identifies a novel protein that negatively affects dNTP pools. Mol Cell 1998;2:329-40.

39. Zhao X, Georgieva B, Chabes A, Domkin V, Ippel JH, Schleucher J, et al. Mutational and structural analyses of the ribonucleotide reductase inhibitor Sml1 define its Rnr1 interaction domain whose inactivation allows suppression of mec1 and rad53 lethality. Mol Cell Biol 2000;20:9076-83.

40. Chabes A, Domkin V, Thelander L. Yeast Sml1, a protein inhibitor of ribonucleotide reductase. J Biol Chem 1999;274:36679-83.

41. Zhang Z, Yang K, Chen CC, Feser J, Huang M. Role of the C terminus of the ribonucleotide reductase large subunit in enzyme regeneration and its inhibition by Sml1. Proc Natl Acad Sci USA 2007; 104:2217-22.

42. Zhao X, Chabes A, Domkin V, Thelander L, Rothstein R. The ribonucleotide reductase inhibitor Sml1 is a new target of the $\mathrm{Mec} 1 / \operatorname{Rad} 53$ kinase cascade during growth and in response to DNA damage. EMBO J 2001;20:3544-53.

43. Zhao X, Rothstein R. The Dun1 checkpoint kinase phosphorylates and regulates the ribonucleotide reductase inhibitor Sml1. Proc Natl Acad Sci USA 2002;99:3746-51.

44. Andreson BL, Gupta A, Georgieva BP, Rothstein R. The ribonucleotide reductase inhibitor, $\mathrm{Sml1}$, is sequentially phosphorylated, ubiquitylated and degraded in response to DNA damage. Nucleic Acids Res 2010;38:6490-501.
45. Uchiki T, Dice LT, Hettich RL, Dealwis C. Identification of phosphorylation sites on the yeast ribonucleotide reductase inhibitor Sml1. J Biol Chem 2004;279:11293-303.

46. Yao R, Zhang Z, An X, Bucci B, Perlstein DL, Stubbe J, et al. Subcellular localization of yeast ribonucleotide reductase regulated by the DNA replication and damage checkpoint pathways. Proc Natl Acad Sci USA 2003;100:6628-33.

47. An X, Zhang Z, Yang K, Huang M. Cotransport of the heterodimeric small subunit of the Saccharomyces cerevisiae ribonucleotide reductase between the nucleus and the cytoplasm. Genetics 2006;173:63-73.

48. Lee YD, Elledge SJ. Control of ribonucleotide reductase localization through an anchoring mechanism involving Wtm1. Genes Dev $2006 ; 20: 334-44$

49. Zhang Z, An X, Yang K, Perlstein DL, Hicks L, Kelleher N, et al. Nuclear localization of the Saccharomyces cerevisiae ribonucleotide reductase small subunit requires a karyopherin and a WD40 repeat protein. Proc Natl Acad Sci USA 2006;103:1422-7.

50. Liu C, Powell KA, Mundt K, Wu L, Carr AM, Caspari T. Cop9/ signalosome subunits and Pcu4 regulate ribonucleotide reductase by both checkpoint-dependent and -independent mechanisms. Genes Dev 2003;17:1130-40

51. Lee YD, Wang J, Stubbe J, Elledge SJ. Dif1 is a DNA-damage-regulated facilitator of nuclear import for ribonucleotide reductase. Mol Cell 2008;32:70-80

52. Wu X, Huang M. Dif1 controls subcellular localization of ribonucleotide reductase by mediating nuclear import of the R2 subunit. Mol Cell Biol 2008;28:7156-67.

53. Sanvisens N, Puig S. Causes and consequences of nutritional iron deficiency in living organisms. In: Biology of starvation in humans and other organisms. In: Merkin TC, editor. Hauppauge, New York: Nova Science Publishers; 2011. p. 245-76.

54. Cavanaugh PF, Jr., Porter CW, Tukalo D, Frankfurt OS, Pavelic ZP, Bergeron RJ. Characterization of L1210 cell growth inhibition by the bacterial iron chelators parabactin and compound II. Cancer Res $1985 ; 45: 4754-9$

55. Furukawa $\mathrm{T}$, Naitoh $\mathrm{Y}$, Kohno H, Tokunaga R, Taketani S. Iron deprivation decreases ribonucleotide reductase activity and DNA synthesis. Life Sci 1992;50:2059-65.

56. Sanvisens N, Bano MC, Huang M, Puig S. Regulation of ribonucleotide reductase in response to iron deficiency. Mol Cell 2011;44:759-69

57. Puig S, Askeland E, Thiele DJ. Coordinated remodeling of cellular metabolism during iron deficiency through targeted mRNA degradation. Cell 2005;120:99-110.

58. Puig S, Vergara SV, Thiele DJ. Cooperation of two mRNA-binding proteins drives metabolic adaptation to iron deficiency. Cell Metab 2008;7:555-64.

59. Netz DJ, Stith CM, Stumpfig M, Kopf G, Vogel D, Genau HM, et al. Eukaryotic DNA polymerases require an iron-sulfur cluster for the formation of active complexes. Nat Chem Biol 2011;8:125-32.

60. Salguero I, Guarino E, Shepherd ME, Deegan TD, Havens CG, MacNeill SA, et al. Ribonucleotide reductase activity is coupled to DNA synthesis via proliferating cell nuclear antigen. Curr Biol 2012;22:720-6

Biomed J Vol. 36 No. 2

March - April 2013 